\title{
Retraction Note to: Nonsense variants in STAG2 result in distinct sex- dependent phenotypes
}

\section{Hiromi Aoi - Ming Lei · Takeshi Mizuguchi • Nobuko Nishioka • Tomohide Goto · Sahoko Miyama • Toshifumi Suzuki (D) Kazuhiro Iwama (D) - Yuri Uchiyama - Satomi Mitsuhashi - Atsuo Itakura • Satoru Takeda • Naomichi Matsumoto}

Published online: 15 June 2020

(c) The Author(s), under exclusive licence to The Japan Society of Human Genetics 2020

Retraction to: Journal of Human Genetics

https://doi.org/10.1038/s10038-019-0571-y

The authors have retracted this article [1] due to a critical error in the reported data. In the published article, Case 1's sex was reported as male. Reexamination of the data showed that Case 1 was in fact female. As the key conclusions of the published article depended on the sex of the cases, the conclusions drawn in the article are invalid. All authors agree to this retraction.

\section{Reference}

1. Aoi H, Lei M, Mizuguchi T, et al. Nonsense variants in STAG2 result in distinct sex-dependent phenotypes. J Hum Genet. 2019;64:487-492. https://doi.org/10.1038/s10038-019-0571-y 